It is in this universal and eternal creed that the charlatan finds his support. In a civilized community the belief in magic is concealed beneath the veneer of education and does not obtrude itself in normal life, but the veneer cracks as soon as any strong emotion is aroused, and both pain and fear of death aro potent causes for arousing such emotions. The exactitudes of science are utterly antipathetic to the sick person, who resents being regarded as a "case" for whom the chances of recovery are so many per thousand. Tho more intelligent turn to religion and to spiritual healers to appeal to some higher power to intervene on their behalf and set aside the soulless laws of nature that are threatening their safety.

The less intelligent revert to the oldest form of belief and seek someone who will make strong magic for them and defeat the evil spirits by some potent charm. This is the feeling to which the quack appeals; he claims to be above the laws of science and to possess some charm for defeating disease of any variety.

The nature of the charm changes with the growth of education. A naked nigger howling to the beat of a tom-tom does not impress a European, and most modern Europeans would be either amused or. disgusted by the Black Mass that was popular in the seventeenth century. To-day some travesty of physical science appears to be the most popular form of incantation. But although the form is changeable yet the underlying belief is unchanging and ineradicable, and $I$ think that the medical profession must face the fact that, although their alliance with science has brought them great material success, yet it has brought its special penaity in that it is ever alienating them to an increasing degree from the fundamental beliefs of the common man.

\section{THE TREATMENT OF BURNS.*}

ALEXANDER. MACLENNAN, M.B., C.M. Honorary Consulting Surgeon to the Royal Infirmary, Stirling, and to Falkirk Infirmary.

Ir is highly advisable from tine to time to review our doctrines on treatment, even the most orthodox. We must be ever on the watch for discoveries in collateral scienco which may be applicable to our own. Many conundrums are awaiting for their solution the preliminary discoveryPasteur must precede Lịster. In the case of burns we are confronted by several problems.

\section{Pain.}

Anyone who has been at all severely burned will at once asscrt that the only problem in burns is the relief of pain, and that in the quickest possible time. I readily agree that it is the most important part of the subject. If this were the only item to be dealt with there would be no difficulty, but our remedies to ensure inmediate mitigation of the disaster must not endanger the future for the victim. Fortunately the perception of pain is limited by exhraustion of the nerve centres, and most of those fatally burned are ominously comfortable during the brief interval between the accident and death.

Children are peculiarly susceptible to injury from burns, and most of us have been shocked by an unexpected fatality from a trivial burn. The much disputed status lymphaticus (in the absence of more precise knowledge) brings an otherwise unwarranted death into line with other injuries. The resistance to toxaemias is in those cases much lowered; the fatty degeneration of the liver frequently found at the necropsy may have some bearing on the fatal issue.

For the majority of patients who recover extensive resort to sedatives is necessary, and it is of great importance that their remote general effects be closely watched. I might suggest that the prolonged administration of chloroform, not as an anaesthetic but as an anodyne, may be less toxic and dangerous than the corresponding amount of morphine. The inhalation of a diluted vapour may be allowed for long periods, and may bo safely carried out by a trustworthy nurse.

The surgeon's visit to a ward containing the victims of burning is evidently a time of trial to the patients; they

* Read in the Section of Surgery at the Annual Meeting of the British Medical Association, Edinburgh, 1927. clamour for chloroform, morphine, and death. I know of no condition which occasions such an accumulation of suffering in a single ward. The reniedies wo employ will require to be many-sided, more than one effect being demanded. For example, while a remedy may be soothing it must not encourage suppuration or discharge. In exceptional instances an extensive burn is tided over the period of initial shock, and the subsequent treatment becomes daily more oncrous. For these patients the continuous bath, where circumstances will permit, is a satisfactory plan; unfortunately in only a few cases can it be mado available. Next to the principle of relieving the pain, the exact nature of the first dressing is of importance. Where bullae are present it is not my practice to drain them, the effused serum being the best covering possible; when they show signs of pus formation they should be emptied. Spraving the area involved with wax solution and covering it with an oily and bland substance are procedures which have rightly found a lasting place in treatment. I would suggest the value of covering the scorched surface with waxed paper; angles may be cut to allow of the discharge reaching the dressing. Such waxed paper may bo sterilized without detriment. A thick tissue paper soaked in carbolic oil may be also similarly used.

There are few cases where good nursing is more-rewarded; extreme gentleness in handling is demanded, and the raw surface should never be wiped with gauze, any discharge being washed off. I firmly believe that the pain associated with a burn, in all but the final stage, is the cause of most of the trouble met with; hence applications which relieve pain will give better results, even remotely. Thus alcohol has achieved some reputation as a suitable application; its effects are not due to its antiseptic action, but to its anaesthetic action on the nerve endings and to its desiccating properties. The possible precipitating effect on toxins can be ignored. In picric acid we have another remedy which is very effective, not altogether because it is an antiseptic, but because it is analgesic, and also because it coagulates albumin. By thus causing the production of a layer of sterile albumin which is at the same time emanating analgesic properties a very good effect is produced. Picric acid is one of the scheduled explosives, and can only be procured in solution $\mathrm{cr}$ in gauze. It may be applied in a saturated solution, but should not be spread too extensively because of its own poisoning properties. Toxicity may be recognized by the onset of fever, accompanied by a rise in pulse rate and the production of a widespread itchy measly rash. The urine becomes green tinted as in carboluria, and there is much general distress. After mitigating the pain future contingencies must be considered.

Shock.

The treatment of this accompanying condition should be conducted along recognized lines. Repeated small doses of opium or morphine with oral administration of alcohol should be given. If alcohol is being extensively applied as a dressing some will be absorbed, so it need not be given otherwise. In a burn the central nervous system is stimulated to the point of exhaustion, and there is no want of explanations for many of the associated phenomena.

\section{The Body Fluids.}

It has been shown that the blood tends to become-at first at any rate-concentrated and less alkaline, as well as to contain more waste products. The symptoms have been explained as being largely due to absorption of substances produced in the tissues by the destructive action of the heat, but this cannot be merely a chemical reaction. Some time ago in attempting to get protein shock I injected the products of partial destruction by heat of animal tissues, but neither intravenous nor intracellular injection caused the slightest effect. It is also affirmed that the chlorides are reduced as in pneumonia, and indeed in many other diseases. A diminution in the chlorides is more usually associated with an alkalosis. From whichever point we view the subject the essential treatment, whether acidosis or alkalosis be present, is the administration of fluid; this may be loaded with alkalis or sodium chloride -I do not think it matters which-provided we introduce fluids into the circulation. 
Some degree of sepsis Sepsis. surface is less able to resist either a fresh invasion or the growth of organisms unscathed by the heat. Powerful antiseptics cannot be applied, nor can weak ones be frequently used. In many cases the attempt to apply a theory may do more harm than good; hence digressions from established authority should be very carefully introduced. I know of no better antiseptic for a burned surface than normal sheep's or horse's serum; the cost is its only drawback. Pneumonia, which accounts for many of the deaths after burns, is probably a manifestation of sepsis, and is produced by a septic embolism.

Duodenal Vlcer.

This is, in my experience, incidentally rarer among the burned than among those not so affected. I have never seen one case in patients suffering from burns. It is more the figment of the imagination of the student going in for an examination.

\section{Surgical Intervention.}

In many cases we do not apply the principles of surgery early enough. At first burned patients are unable to sustain further inroad into their vitality, and later on it is sometimes forgotten that surgery may improve the outlook, both as regards immediate morbidity and ultimate appearance. Where a limb has been almost denuded of skin carly amputation is indicated. Short of this it will be found in many cases-and this is the experience of most of us-that an ulcer produced by surgery is much less severe than one resulting from $\dot{a}$ burn. It is the uncertainty of our judgement regarding the vitality of any section of burned surface which makes us hesitate to resort to extensive excisions. It is often surprising how many islets of epithelium appear among the granulations after the slough of what we considered to be the entire thickness of skin has separated. Even though we may finally have larger areas to epithelialize than would have formed without excision we certainly lessen suffering very materially. I think the tendency hitherto has been conservative. Simultaneously with the excision provision should be made for grafting. Large ulcers are to be treated upon general lines; the longer they remain the greater will be the tendency for contractures to occur. On the other hand, acquiescing in the production of a contracture may result in a rapid obliteration of the ulcer, yet such a contracture should not be allowed to develop, but the ulcer should be healed by other means.

\section{Grafting}

Successful grafting requires careful preparation of the field; indeed, until the septic process has ended grafting should not be undertaken if the Thiersch or Wolff method be adopted. Many years ago I introduced a method of grafting to which the name of "tunnel" grafting was given; it can be applied at an earlier stage than any cther, and has a high percentage of successes. Narrow grafts are embedded under the surface and their locality marked by laying alongside them suture threads. After allowing for their taking root, which occurs in about ten days, the covering surface is removed so as to expose the grafts completely. On account of the close resemblance of the granulation tissue developed to sarcomatous tissue in its destructive effects a thin epithelial covering is almost certain of being destroyed from time to time. This feature of constantly recurring ulceration must be familiar to all; hence a solid covering is essential if healing is to be permanent. It is interesting to note that if embedded grafts are left for any length of time under or in the granulations they entirely disappear. This is a usual phenomenon, otherwise operation implantation grafts would be commoner.

As an aid to the spread of epithelium scarlet-red applied in an ointment is a very decided stimulant. Where serious grafting is required the pedicled graft is best; it is often noticeable that sepsis tends, when these grafts are freed, to light up the processes of ulceration. This development sometimes is the cause of failure in grafting for the relief of contracture even in cases long since healed. It would seem that cicatricial tissue is capable of retaining, encysted within its meshes, infection in an active state. The development of keloid is one evidence of such a power.

In considering burns generally we nearly always have in our minds the effects of heat; yet cold, chemicals, ultiaviolet rays in sunlight direct or reflected from snow, or those produced electrically, induce similar disturbances. Another form of burn, fortunately now less common, is that produced by radium and $x$ rars. Burns so caused show great lesistance to healing, and their tendency to become malignant is well recognized. Occasionally when an ordinary burn has been allowed to pass into a chronic ulcer malignancy may become engrafted.

\section{Discussion.}

Mr. W. C. Wilson (Edinburgh) gare his experiences with tannic acid in the treatment of 37 cases of burns in children. He said that at the stage of initial shock tannic acid was beneficial in that it promoted a rapid and complete analgresia. In the stage of acute toxaemia the main indication was to prevent absorption of the toxin; in this respect the action of tannic acid was striking and valuable, for by coagulating the tissues at the burnt area it prevented absorption of toxin into the blood stream. In the stage of septic toxaemia tannic acid, by providing a firm, dry covering to the wound, minimized the incidence of sepsis; it also protected the growing epithelium and promoted rapid healing. Primary shock was treated in the usual way--a small dose of morphine being given if necossary. No general anaesthesia was employed. The burnt areas were cleansed rapidly with ether. A fresh!y prepared solution of $2 \frac{1}{2}$ per cent. of tannic acid in warm sterile water was sprayed over the burnt areas at intervals of an hour, and the application was dried by electrical heat. This was continued until a brownish-black coagulum formed (eight to twelve times). The bed-clothes "were cradled and the parts exposed to the air during this time and until the coagulum was removed. While spraying the face, the eves, ears, and nostrils must be protected. Slides were shown to illustrate the effect of treatment on mortality, the prevention of acute toxaemia, and the improvement in prognosis thus obtained.

Mr. Sreward (London) agreed that tamnic acid gave excellent results. He proferred to make a careful toilet under anaesthesia at the beginning of treatment.

Mr. R. M. Manwaring-White (Northwich) said that the three essentials of relief of pain, treatment of shock, and prevention of sepsis were obtained by using the pommade of Reclus, which contained phenazone, salol, boric acid, iodoform, phenol, mercury perchloride, and raseline.

\section{A CASE OF ABNORMAL FAT METABOLISM. \\ BY}

JAMES A. TORRENS, M.D., F.R.C.P., PHYSICIAN, ST. GEORGE'S HOSPITAL

H. Gatisborough, M.B., M.R.C.P., AsSISTANT PHYSICIAN, ST. GzORGE's hOSPITAL; AYD

J. A. GARDNER, M.A., READER IN PHYSIOUOGICAL CHEMISTRY, UNIVERSITY OF LONDON.

THe clinical symptoms in the following unusual case suggested some defect in fat metabolism, and in the hope of ascertaining its nature a thorough investigation was made; the results, though they did not explain the mystery, seem worthy of record.

A girl, aged 13, was sent to hospital because her mother had A girl, aged 13, loss of weight for two years; she had not noticed progressive loss of weight for two years; she had not
seemed ill; her appetite was uniformly good; her bowels were open naturally, and she was quite ordinarily energetic, though during the last the child various tonics, and had persistently tried to had given the child various tonics, and had oil; these extra fatty feed her up with cream, milk, and cod-liver on, these extra fatty foods were taken readily, and hation had not started. There had been no previous illness of note. 